\title{
OPTIMAL PORTFOLIO WITH A WAGE INCREASE
}

\author{
Ho-SEOK LEE*
}

\begin{abstract}
In this paper, we solve the optimal investment problem when the investor gets labor income and may have a wage increase. We assume the investor has constant absolute risk aversion(CARA) utility and obtain closed form solutions.
\end{abstract}

\section{Introduction}

In Merton [4], the optimal investment and consumption policies of an investor in a continuous time were investigated by using dynamic programming principle. It is assumed that the investor's portfolio consists of the risk free asset(bank account) and the risky asset which is assumed to follow a geometric Brownian motion. In this paper, we assume that the investor has a chance to get a wage increase before retirement time $T$. We consider two cases. First, the time of wage increase is a predetermined time $T_{1}<T$. Second, investor gets a wage increase at a random time $\tau$.

\section{The model}

Let us consider a portfolio composed of a risk free asset with rate of return $r$ and a risky asset with the dynamics(geometric Brownian motion)

$$
\frac{d p_{t}}{p_{t}}=\mu d t+\sigma d B_{t}
$$

where $B_{t}$ is a standard Brownian motion on a probability space $(\Omega, \mathcal{F}, P)$. Let $\left\{\mathcal{F}_{t}\right\}_{t \geqslant 0}$ be an augmentation of the natural filtration generated by two independent random variables $B_{t}(t \geqslant 0)$ and a random time $\tau$.

Received April 23, 2013; Accepted July 08, 2013.

2010 Mathematics Subject Classification: Primary 91G10.

Key words and phrases: portfolio optimization, CARA utility, Hamilton-JacobiBellman equation. 
From Merton [4], if there is no labor income, the wealth process is given by $d W_{t}=\left[r W_{t}+\pi_{t}(\mu-r)\right] d t+\sigma \pi_{t} d B_{t}$, where $\pi_{t}$ is amount of investment in risky asset and the value function is given by

$$
V_{0}(W, t)=\max _{\left\{\pi_{s}, t \leqslant s \leqslant T\right\}} E\left[U\left(W_{T}\right) \mid \mathcal{F}_{t}\right], W_{t}=W .
$$

$U(\cdot)$ is the utility function and we assume that $U(x)=-\frac{1}{\eta} e^{-\eta x}$ with $\eta>0$. Then,

$$
V_{0}(W, t)=-\frac{1}{\eta} e^{-\eta W e^{r(T-t)}} e^{-\frac{(\mu-r)^{2}}{2 \sigma^{2}}(T-t)} .
$$

The optimal portfolio in risky asset is

$$
\pi^{*}=\frac{\mu-r}{\eta \sigma^{2} e^{r(T-t)}}
$$

If the investor gets labor income, $d W_{t}=\left[r W_{t}+\pi(\mu-r)+Y_{t}\right] d t+\sigma \pi_{t} d B_{t}$, where $Y_{t}$ is labor income rate. It is not rational that the investor gets his wage at constant rate all the time while he is working. Rather, it is reasonable to assume that working people sometimes has chances to have a wage increase in the event of promotion, etc. We assume that the investor has one chance of income increment over the horizon $T$, which is the fixed time of retirement. Let us consider two different cases:

Case(1) With finite horizon $T$, investor gets an increment in income rate at a predetermined time $T_{1}<T$. In this case, $Y_{t}=\varepsilon$ for $0 \leqslant t<T_{1}$, $Y_{t}=\varepsilon+\delta$ for $T_{1} \leqslant t \leqslant T$. This situation can be interpreted as follows. An employer and an employee make a contract that employee is guaranteed to get an increase in income at a predetermined time $T_{1}$.

Case(2) With finite horizon $T$, investor gets an increment in income rate at a random time $\tau$, for example a stochastic variable with exponential type distribution. In this case $Y_{t}=\varepsilon$ for $0 \leqslant t<\tau, Y_{t}=\varepsilon+\delta$ for $\tau \leqslant t$. Suppose that an employee wants to get his wage on his ability, so he prefers to obtain increase in income at an uncertain time. This time, of course, is assumed to be earlier if an employee is of more ability. But his efficiency is not deterministic because of accidents, sickness, etc. Possibly, the investor could not have income increase before $T$.

If an employee could choose one of the two types of income stated above and he seeks to maximize expected utility of terminal wealth, which one should he choose? Assume the utility function is of constant absolute 
risk aversion (CARA) type, i.e.,

$$
U(x)=-\frac{1}{\eta} e^{-\eta x} \text { with } \eta>0 .
$$

\section{Determination of value functions}

\subsection{Case(1): Predertermined jump time of income}

We consider

$$
V(W, t)=\max _{\left\{\pi_{s}, t \leqslant s \leqslant T\right\}} E\left[U\left(W_{T}\right) \mid \mathcal{F}_{t}\right]
$$

with

(3.2) $d W_{t}=\left[r W_{t}+\pi(\mu-r)+Y_{t}\right] d t+\sigma \pi_{t} d B_{t}, Y_{t}=\varepsilon+\delta 1_{\left\{T_{1} \leqslant t \leqslant T\right\}}$,

where $1_{\{\cdot\}}$ is an indicator function. We determine $V(W, t)$ for $T_{1} \leq t \leq T$ first and find $V(W, t)$ for $0 \leq t<T_{1}$ later. For this, let

$$
V(W, t) \equiv \begin{cases}V^{1}(W, t, \delta), & 0 \leq t<T_{1} \\ V^{2}(W, t, \delta), & T_{1} \leq t \leq T\end{cases}
$$

Theorem 3.1. The value function $V(W, t)$ is given by

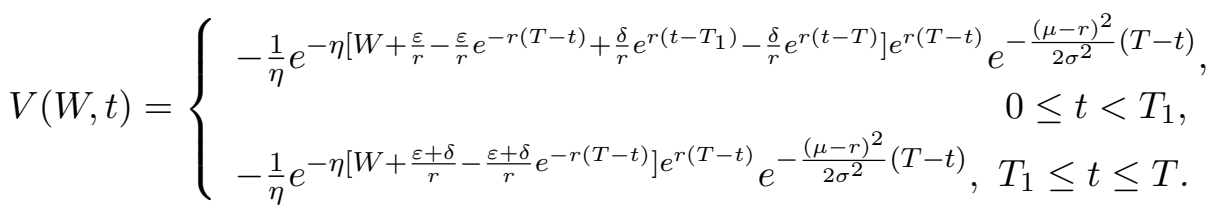

In this case, the optimal portfolio in the risky asset is also given by

$$
\pi^{*}=\frac{\mu-r}{\eta \sigma^{2} e^{r(T-t)}},
$$

for all $0 \leq t \leq T$.

Proof. The certainty equivalent present value of labor income, $P(Y, t)$ is given by

$$
E \int_{t}^{T} \zeta_{u} Y_{u} d u
$$

where $\zeta_{u}=e^{-r(u-t)-\frac{1}{2}\left(\frac{\mu-r}{\sigma}\right)^{2}(u-t)-\left(\frac{\mu-r}{\sigma}\right)\left(B_{u}-B_{t}\right)}\left(\right.$ see Koo [2]). $V^{2}(W, t, \delta)$ is obtained by substituting $W$ in (2.1) with modified initial wealth $W+$ $P(Y, t)$. Hamilton-Jacobi-Bellman equation for $V^{1}(W, t, \delta)$ is given by

$$
V_{t}^{1}+(r W+\varepsilon) V_{W}^{1}+\max _{\pi}\left[\pi(\mu-r) V_{W}^{1}+\frac{1}{2} \sigma^{2} \pi^{2} V_{W W}^{1}\right]=0 .
$$


FOC implies $\pi^{*}=-\frac{\mu-r}{\sigma^{2}} \frac{V_{W}^{1}}{V_{W W}^{1}}$. With this $\pi^{*},(3.3)$ becomes

$$
V_{t}^{1}+(r W+\varepsilon) V_{W}^{1}-\frac{(\mu-r)^{2}}{2 \sigma^{2}} \frac{\left(V^{1}\right)^{2}}{V_{W W}^{1}}=0 .
$$

$V^{1}(W, t, \delta)$ should have the no bubble condition

$$
\lim _{\delta \rightarrow 0} V^{1}(W, t, \delta)=V^{2}(W, t, 0)
$$

and the following value matching condition at $t=T_{1}$

$$
V^{1}\left(W, T_{1}, \delta\right)=V^{2}\left(W, T_{1}, \delta\right)
$$

(We can think of $\delta 1_{\left\{T_{1} \leqslant t \leqslant T\right\}}$ as some kind of flow payoffs. Miao and Wang [3] consider lump-sum and flow payoffs and present no bubble conditions, value matching conditions at the time when one of two above payoffs types is provided.). To slove (3.4) with (3.5), (3.6), conjecture $V^{1}(W, t, \delta) \equiv-\frac{1}{\eta} e^{-\eta\left[W+\frac{\varepsilon}{r}-\frac{\varepsilon}{r} e^{-r(T-t)}+\delta A(t)\right] e^{r(T-t)}} e^{-\frac{(\mu-r)^{2}}{2 \sigma^{2}}(T-t)}$. After substituting this conjectured form into (3.4), subject to (3.5), (3.6), we get

$$
A(t)=\frac{1}{r}\left[e^{r\left(t-T_{1}\right)}-e^{r(t-T)}\right] .
$$

So, we obtain $V^{1}(W, t, \delta)$ and the optimal portfolio $\pi^{*}$.

\subsection{Case(2): Random jump time of income}

Let us introduce probability structure of our jump process. Let $f(t) h+o(h)$ be the probability of income jump in $(t, t+h)$ with $f(t)$ containing its own parameters . Denote our jump time by $\tau$. Then $\tau$ has cumulative distribution function

$$
F(s)=\operatorname{prob}\{\tau<s\}=\int_{0}^{s} f(t) d t .
$$

Let us consider an artificial random variable $x(t)$ such that $x(t)=0$ for $0 \leqslant t<\tau, x(t)=1$ for $\tau \leqslant t \leqslant T$. Our value function $J(W, t, x)$ is given by

$$
J(W, t, x)=\left\{\begin{array}{l}
J(W, t, 0)=\max _{\left\{\pi_{s}, t \leqslant s<\tau\right\}} E\left[U\left(W_{T}\right) \mid \mathcal{F}_{t}\right], 0 \leq t<\tau, \\
J(W, t, 1)=V(W, t), \tau \leq t \leq T
\end{array}\right.
$$

with

$$
d W_{t}=\left[r W_{t}+\pi(\mu-r)+\varepsilon+\delta 1_{\{\tau \leqslant t \leqslant T\}}\right] d t+\sigma \pi_{t} d B_{t}
$$

and

$$
V(W, t)=-\frac{1}{\eta} e^{-\eta\left[W+\frac{\varepsilon+\delta}{r}-\frac{\varepsilon+\delta}{r} e^{-r(T-t)}\right] e^{r(T-t)}} e^{-\frac{(\mu-r)^{2}}{2 \sigma^{2}}(T-t)} .
$$


Theorem 3.2. $J(\cdot, \cdot, 0)$ is given by

$$
J(W, t, 0)=-\frac{1}{\eta} e^{-\eta W e^{r(T-t)}} b(t),
$$

where $b(t)=e^{\int_{T}^{t} p(u) d u}\left[-\int_{T}^{t} q(u) e^{-\int_{T}^{u} p(v) d v} d u+1\right]$ with

$$
\begin{aligned}
& p(t)=f(t)+\eta \varepsilon e^{r(T-t)}+\frac{(\mu-r)^{2}}{2 \sigma^{2}}, \\
& q(t)=f(t) e^{-\eta\left[\frac{\varepsilon+\delta}{r} e^{r(T-t)}-\frac{\varepsilon+\delta}{r}\right]-\frac{(\mu-r)^{2}}{2 \sigma^{2}}(T-t)},
\end{aligned}
$$

and the optimal portfolio in the risky asset is given by

$$
\pi^{*}=\frac{\mu-r}{\eta \sigma^{2} e^{r(T-t)}}
$$

Proof. Henceforth, we will denote $J(W, t, 0)$ as $J(W, t) \equiv J$. By principle of optimal stochastic control, Hamilton-Jacobi-Bellman equation is given by

$$
\begin{aligned}
J_{t}+ & f(t)[J(W, t, 1)-J]+(r W+\varepsilon) J_{W}+ \\
& \max _{\left\{\pi_{s}, t \leqslant s<\tau\right\}}\left[\pi(\mu-r) J_{W}+\frac{1}{2} \sigma^{2} \pi^{2} J_{W W}\right]=0
\end{aligned}
$$

for $0 \leq t<\tau$. (For detail derivation of (3.7), see Kushner [1].) That is

$$
\begin{array}{r}
J_{t}-\frac{1}{\eta} f(t) e^{-\eta\left[W+\frac{\varepsilon+\delta}{r}-\frac{\varepsilon+\delta}{r} e^{-r(T-t)}\right] e^{r(T-t)}} e^{-\frac{(\mu-r)^{2}}{2 \sigma^{2}}(T-t)}-f(t) J \\
+(r W+\varepsilon) J_{W}-\frac{(\mu-r)^{2}}{2 \sigma^{2}} \frac{J_{W}^{2}}{J_{W W}}=0 .
\end{array}
$$

To solve (3.8), conjecture $J(W, t)=-\frac{1}{\eta} e^{A(t) W+B(t)}, A(t)$ and $B(t)$ are functions of $t$ only. Substituting this form of $J(W, t)$ into (3.8), we get $A(t)=-\eta e^{r(T-t)}$ and an ordinary differential equation of $B(t)$ as follows:

$$
\begin{aligned}
& e^{B(t)}\left[B^{\prime}(t)-f(t)-\eta \varepsilon e^{r(T-t)}-\frac{(\mu-r)^{2}}{2 \sigma^{2}}\right] \\
& \quad+f(t) e^{-\eta\left[\frac{\varepsilon+\delta}{r} e^{r(T-t)}-\frac{\varepsilon+\delta}{r}\right]-\frac{(\mu-r)^{2}}{2 \sigma^{2}}(T-t)}=0 .
\end{aligned}
$$


Let $e^{B(t)} \equiv b(t)$, then (3.9) becomes $b^{\prime}(t)-p(t) b(t)+q(t)=0$, where

$$
\begin{aligned}
& p(t)=f(t)+\eta \varepsilon e^{r(T-t)}+\frac{(\mu-r)^{2}}{2 \sigma^{2}}, \\
& q(t)=f(t) e^{-\eta\left[\frac{\varepsilon+\delta}{r} e^{r(T-t)}-\frac{\varepsilon+\delta}{r}\right]-\frac{(\mu-r)^{2}}{2 \sigma^{2}}(T-t)} .
\end{aligned}
$$

and we get

$$
b(t)=e^{\int_{T}^{t} p(u) d u}\left[-\int_{T}^{t} q(u) e^{-\int_{T}^{u} p(v) d v} d u+1\right]
$$

such that $J(W, T)=-\frac{1}{\eta} e^{-\eta W}$. So, our value function for $0 \leq t<\tau$ is

$$
J(W, t)=-\frac{1}{\eta} e^{-\eta e^{r(T-t)} W} b(t)
$$

and the optimal portfolio is given by (for $0 \leq t \leq T$ )

$$
\pi^{*}=-\frac{\mu-r}{\sigma^{2}} \frac{J_{W}}{J_{W W}}=\frac{\mu-r}{\eta \sigma^{2} e^{r(T-t)}} .
$$

\section{References}

[1] H. J. Kushner, Stochastic Stability and Control, Academic Press, New York, 1967.

[2] H. K. Koo, Consumption and Portfolio Choice with Uninsurable Income Risk, PhD Dissertiation, 1992.

[3] J. Miao and N. Wang, Investment, Consumption and Hedging under Incomplete Markets, Preprint.

[4] R. C. Merton, Optimum Consumption and Portfolio Rules in a ContinuousTime Model, J. Econ. Theory 3 (1971), 373-413.

$*$

FX \& Derivatives Trading Div.

Korea Exchange Bank

Seoul 100-793, Republic of Korea

E-mail: kaist.hoseoklee@gmail.com 